\title{
SYNTHESIS AND CHARACTERIZATION OF CdSe COLLOIDAL QUANTUM DOTS IN ORGANIC SOLVENT
}

\author{
Ion Geru ${ }^{\mathrm{a}}$, Olga Bordianª, , Constantin Loshmanskyc, Ion Culeac ${ }^{\mathrm{a}, \mathrm{b}}$, Constantin Turta ${ }^{\mathrm{a}}$ \\ anstitute of Chemistry of Academy of Sciences of Moldova, 3, Academiei str., Chisinau MD 2028, Republic of Moldova \\ ${ }^{b}$ Institute of Applied Physics of Academy of Sciences of Moldova, 5, Academiei str., Chisinau MD 2028, Republic of Moldova \\ ${ }^{c}$ University of the Academy of Sciences of Moldova, 3/2, Academiei str., Chisinau MD 2028, Republic of Moldova \\ *e-mail: iongeru11@gmail.com
}

\begin{abstract}
In this paper we present experimental results on preparation and characterization of colloidal CdSe quantum dots in organic solvent. CdSe QDs were synthesized following a modified literature method. CdSe QDs were isolated by adding acetone to the cooled solution followed by centrifugation. CdSe QDs have been characterized by UV-Vis absorption and photoluminescent (PL) spectroscopy. The average CdSe particles size estimated from the UV-Vis absorption spectra was found to be in the range 2.28-2.92 nm which is in good agreement with PL measurements.
\end{abstract}

Keywords: colloidal quantum dots, semiconductor, cadmium selenide, nanocrystals, photoluminescence.

\section{Introduction}

Among various semiconductor quantum dots (QDs) extensive research efforts have been developed over the last decade in the case of CdSe QDs because of their attractivity for various application in optoelectronics, photonics, medicine, etc. [1-3]. In spite of the problems related to the toxicity of Cd compounds, the possibility of tuning of the optical parameters of CdSe QDs makes them very attractive for technological development as well as for optoelectronics and biomedical applications. The size of QDs can be easily controlled by different ways, for example by variation of the reaction time, or the temperature of the solvents, by variation of the concentration of the reaction solvents, etc. This means a relatively simply and convenient technology for controlling the optical parameters of the nanocompozite structures for practical exploitation. For example, PL emission from colloidal CdSe quantum dots can be adjusted in a relatively wide spectral range from 465 to $670 \mathrm{~nm}$ [2]. The technology makes it possible to prepare different sized nanocrystals with high PL efficiency and narrow PL band, while PL emission may cover a narrow spectral band, tuned from blue to read and even up to near infrared. Control and improvement of optical properties of CdSe quantum dots remain on the agenda of research in the field of nanotechnology.

\section{Experimental details}

The technology of preparation of the CdSe nanodots is well described in details in the literature and an extended list of publications in this field can be consulted. Colloidal semiconductor QDs described in this paper were prepared via a chemical route.

\section{Materials}

Cadmium oxide (99.999\%), oleic acid (90\%), selenium (99.5\%, 100 mesh), trioctylphosphine (90\%), 1-octadecene (90\%), tributylphosphine (90\%), were purchased from Aldrich and used without further purification. Synthesis

CdSe semiconductor nanocrystals were synthesized in a three-neck flask equipped with condenser, magnetic stirrer, thermocouple, and heating mantle. Typically the synthesis of the nanocrystals CdSe was carried out at $170^{\circ} \mathrm{C}$ by the following method. At the first step the stock solution was prepared, containing $180 \mathrm{mg}$ of Se powder, $3 \mathrm{~mL}$ of 1-octadecene (ODE), $2 \mathrm{~mL}$ tributulphosphine (TBP) and $0.8 \mathrm{~mL}$ of trioctylphosphine (TOP) are mixed with intense stirring. The Cd precursor solution was prepared from $80 \mathrm{mg}$ of CdO powder, $3 \mathrm{~mL}$ of 1-octadecene, $1 \mathrm{~mL}$ of TBP (tributulphosphine) and $4 \mathrm{~mL}$ of oleic acid. These compositions were mixed together by vigorous stirring. At the next step at the temperature $170{ }^{\circ} \mathrm{C} 2 \mathrm{~mL}$ of the Stocks solution was quickly injected into the hot Cd precursor solution and then this mixture was kept at $170{ }^{\circ} \mathrm{C}$. Different-sized QDs were obtained varying the reaction time. The colloidal quantum dots solutions were collected at determined reaction time interval after the injection, for obtaining the desired size of quantum dots. The obtained CdSe QDs were further isolated and purified by adding acetone to the cooled solution followed by centrifugation at $8000 \mathrm{rpm}$ for about $30 \mathrm{~min}$.

\section{Characterization}

CdSe nanoparticles have been characterized by UV-Vis absorption and photoluminescent (PL) spectroscopy. The average CdSe particles size for three selected samples was estimated from the position of the first excitonic peak in the UV-Vis absorption spectrum and was found to be in the range 2.28 - $2.92 \mathrm{~nm}$ which correlates with PL experimental data. PL spectra were measured under excitation of a laser beam 337 or $405 \mathrm{~nm}$ using a MDR-23 monochromator and a photon counting module H9319-12 connected to a PC. 


\section{Results and discussion}

Figure 1 shows the image of the prepared CdSe quantum dots dispersed in chloroform. From right to left the particle size increases, as well as the suspension color changes from light orange to reddish. This is a good illustration of the effect of the particle size on optical absorption of the quantum dots.

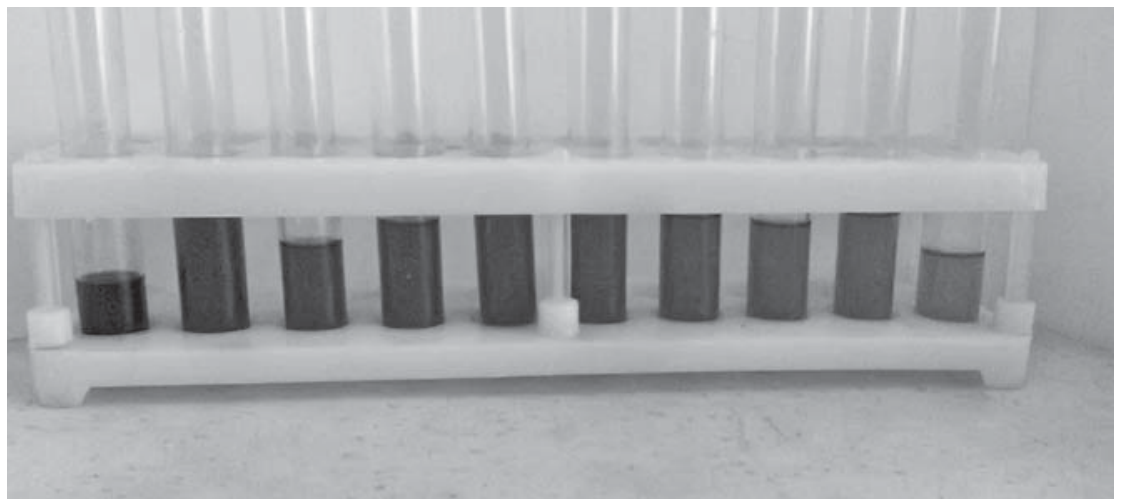

Figure 1. Size-dependent color of luminescent CdSe naoparticles. The particle size increases from right to left.

Figure 2 illustrates the absorbance spectra of the colloidal chloroform solution of the prepared QDs with different particles size. The absorption peaks appear very clear on each curve, indicating the presence of CdSe QDs. The reaction time for selected samples 1, 2, 3 increases by $50 \mathrm{~s}$, and the absorption peak red-shifts from 494.7 up to $543 \mathrm{~nm}$, sequentially. The absorption edge is relatively sharp, indicating a relatively narrow size distribution of the nanoparticles.

From the peak positions, one can estimate the average size of CdSe QDs. From the position of the first excitonic peak the size of nanoparticles have been evaluated through the empirical relation [3]:

$$
d=\left(1.6122 \times 10^{-9}\right) \lambda^{4}-\left(2.6575 \times 10^{-6}\right) \lambda^{3}+\left(1.6242 \times 10^{-3}\right) \lambda^{2}-(0.4277) \lambda+41.57
$$

where $d(\mathrm{~nm})$ is the average diameter of the CdSe QDs and $\lambda(\mathrm{nm})$ is the wavelength of the first excitonic peak of the corresponding sample.

The size of CdSe quantum dots was varied for samples 1 - 3 by changing the reaction time, and was calculated as 2.28, 2.42 and $2.92 \mathrm{~nm}$ respectively. This QDs size implies the strong confinement of the charge carriers, while the confinement energies of the electron and hole are larger than the Coulomb interaction [3-5].

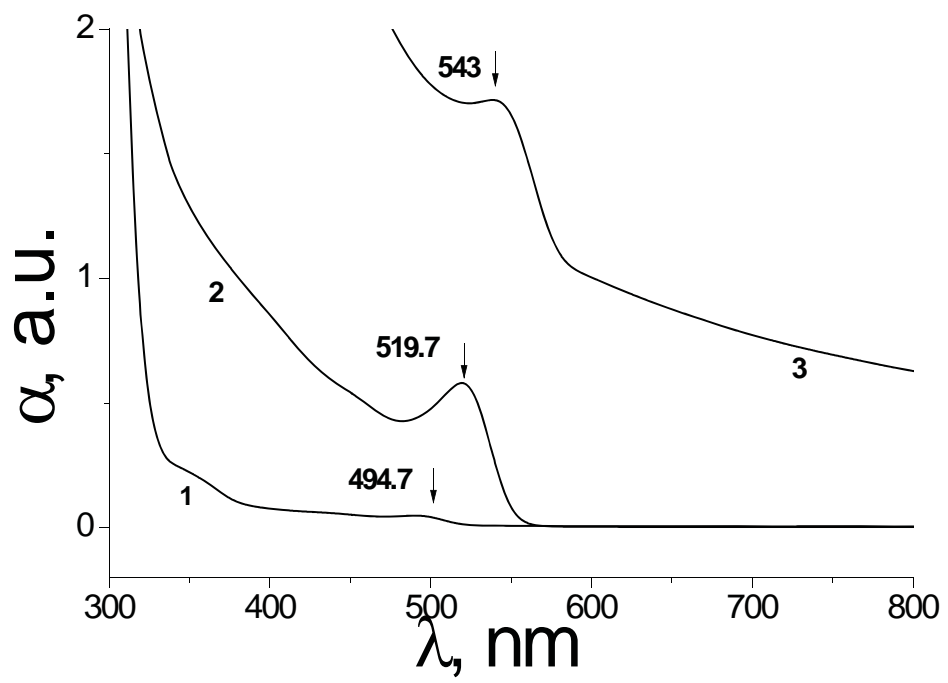

Figure 2. UV-Vis absorption spectra of suspension of CdSe quantum dots in chloroform grown at various reaction times. The plots 1 - 3 correspond to three dots samples collected at $50 \mathrm{~s}$ time interval from the injection. The reaction time, $t:(1) t_{0}$; (2) $t_{0}+50 \mathrm{~s}$; (3) $t_{0}+100 \mathrm{~s}$. 
We can compare the energy of the main excitonic peak $E$ as a function of the size of the nanocrystal from the relation [5-7]:

$$
E=E_{g}+\frac{\hbar^{2} \pi^{2}}{2 R^{2}}\left(\frac{1}{m_{e}^{*}}+\frac{1}{m_{h}^{*}}\right)-\frac{1}{R}\left(\frac{1.8 e^{2}}{4 \pi \varepsilon_{0} \varepsilon_{r}}\right)
$$

where $E_{g}$ is the energy gap of bulk CdSe $\left(\mathrm{E}_{\mathrm{g}}=1.7 \mathrm{eV}, R\right.$ is the size of the nanoparticle, $m_{e}{ }^{*}$ and $m_{h}{ }^{*}$ are the effective masses of the electron and hole. The corresponding values for the effective masses are as reported elsewhere [5,8]: $\frac{m_{e}^{*}}{m_{0}}=0.13$ and $\frac{m_{h}^{*}}{m_{0}}=0.45$ and the dielectric constant $\varepsilon_{r}=10.6, \varepsilon_{0}$ is the permittivity of free space and $e$ is the electron charge. If we take the particles diameter calculated from the absorption spectra in Figure 2 as $2.28 \mathrm{~nm}(1), 2.42 \mathrm{~nm}(2)$ and $2.92 \mathrm{~nm}$ (3), then the calculated exciton energies are respectively 2.31, 2.24 and $2.05 \mathrm{eV}$. On the other side, the positions of the excitonic peaks determined from the absorption spectra in Figure 2 are a bit higher, and represent 2.5, 2.39 and $2.28 \mathrm{eV}$ respectively. The difference in the values of $E$ calculated from (2) and the values determined from the absorption spectra in Figure 2 is determined basically by the error related to Eq.(1) as well as by approximations involved in the theoretical model based on the effective mass approximation with the electron-hole pair confined at the center of an infinite spherical potential wall [5,7].

The energy values calculated from (2) and Figure 2 are higher than $E_{g}$ of the bulk CdSe $(1.7 \mathrm{eV}$ at $300 \mathrm{~K})$ which is indicative of a blue shift of the absorption edge. The increase of the band gap is determined by the quantum size effect of small crystallites, and the calculated values for the diameter of the nanocrystals are less than the bulk CdSe exciton Bohr radius $5.6 \mathrm{~nm}[9,10]$. As far as the size of quantum dots investigated here are smaller than their Bohr radius, they exhibit size-dependent properties [3,6]. Eq.(2) illustrates the quantum confinement effect of quantum dots. In this equation the second term in represents the quantum confinement term which is proportional to $R^{-2}$, and shifts $E$ to higher energy when the diameter of the nanoparticle decreases. The third term in Eq.(2) represents the Coulomb term, which is proportional to $R^{-1}$. It shifts $E$ to lower energy as the radius of the nanoparticles decreases. Consequently, when the particles size decreases the energy gap increases [3,6,7].

The PL emission spectra have been registered at room temperature under the excitation of laser beam $405 \mathrm{~nm}$ (Figure 3). The PL emission spectra of CdSe colloidal QDs solution in chloroform are represented in Figure 3, which shows a Stocks red-shift for each sample. From these spectra one can suggest that for moderate reaction times there one can obtain good PL characteristic with narrow emission peaks. While at longer reaction times, the PL intensity is substantially reduced. We can suggest that at moderate growth times one can obtain better emission characteristics and monodispersed size distribution.

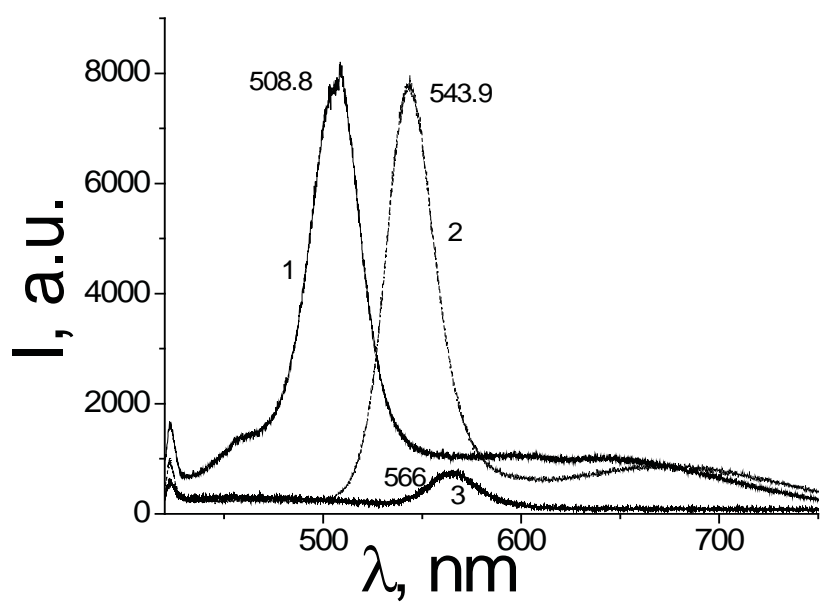

Figure 3. PL spectra of CdSe colloidal quantum dot samples dissolved in chloroform, collected at 50 sec time interval. The plots 1 - 3 correspond to three dots samples, as indicated in Figure 2. The excitation light is a $N_{2}$ laser beam $337 \mathrm{~nm}$.

These experimental results are consistent with previous work on CdSe QDs [4, 8-10]. The PL spectrum for CdSe nanodots can be characterized by an almost Gaussian line with the peak maximum shifting from $508 \mathrm{~nm}$ to $566 \mathrm{~nm}$ in dependence of the CdSe nanoparticle size. The PL emission of CdSe QDs is dominated by a near-band-edge emission; while a weak broad band in the near IR can be related to the surface shallow trap emission is markedly suppressed. The 
PL spectrum for the sample 2 can be deconvoluted in a major Gaussian line, positioned at 508 nm and other two low intensities bands shifted toward red wavelengths (Figure 4). While the CdSe QDs size tends to increase with increasing the reaction time, the PL bands shifts from 508 to $566 \mathrm{~nm}$ (Figure 3), which correlates with the position of the main excitonic peak shifting in the absorption spectrum (Figure 2). The narrow line width of the main PL band suggests that particles have a narrow size distribution, while the low intensity emission from surface trap states at longer wavelengths suggests low concentration of surface defects.

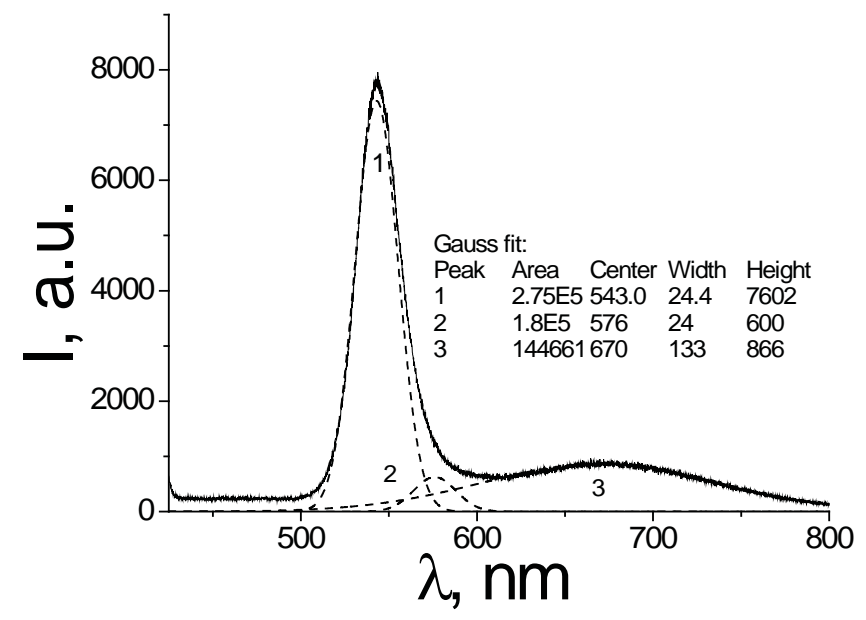

Figure 4. Deconvolution of the PL spectrum for sample 2.

The peaks are situated at 543, 576 and $670 \mathrm{~nm}$.

The FWHM for the main band at 543 is $~ 24 \mathrm{~nm}$.

\section{Conclusions}

We present experimental results on preparation and characterization of colloidal CdSe quantum dots. CdSe nanoparticles were synthesized following a modified literature method. CdSe QDs were isolated by adding acetone to the cooled solution followed by centrifugation. CdSe QDs have been characterized by UV-Vis absorption and photoluminescent (PL) spectroscopy. The average CdSe QDs size estimated from the UV-Vis absorption spectrum was found to be in the range 2.28-2.92 nm which is in good agreement with PL emission spectra registered at room temperature.

\section{References}

1. Somers, R.C.; Bawendi, M.G.; Nocera, D.G. CdSe nanocrystal based chem-/bio- sensors, Chemical Society Reviews, 2007, 36, pp. 579-591.

2. Jorge, P.; Martins, M.A.; Trindade, T.; Santos, J.-L.; Farahi, F. Optical Fiber Sensing Using Quantum Dots, Sensors, 2007, 7, pp. 3489-3534.

3. Yu, W.W.; Qu, L.; Guo, W.; Peng, X. Experimental Determination of the Extinction Coefficient of CdTe, CdSe, and CdS Nanocrystals, Chemistry of Materials, 2003, 15, pp. 2854-2860.

4. Nguyen, H.Q. Synthesis and optical properties of CdSe nanocrystals and CdSe/ZnS core/shell nanostructures in non-coordinating solvents, Advances in Natural Sciences: Nanoscience and Nanotechnology, 2010, 1, doi:10.1088/2043-6254/1/2/025004.

5. Bawendi, M.G.; Steigerwald, M.L. and Brus, L.E. The Quantum Mechanics of Larger Semiconductor Clusters (“Quantum Dots”), Annual Review of Physical Chemistry, 1990, 41, pp. 477-496.

6. Wang, Y.; Herron, N. Nanometer-Sized Semiconductor Clusters: Materials Synthesis, Quantum Size Effects, and Photophysical Properties, The Journal of Physical Chemistry, 1991, 95, pp. 525-532.

7. $\quad$ Brus, L. Quantum crystallites and nonlinear optics, Applied Physics A, 1991, 53(6), pp. 465-474.

8. Biswas, A.and Ghosal, A. Hole Transport Characteristics of CdSe Single Quantum Wells at Low Temperatures, Journal of Electron Devices, 2011, 10, pp. 444-447.

9. Meulenberg, R.W.; Lee, J.R.I.; Wolcott, A.; Zhang, J.Z.; Terminello L.J.; van Buuren, T. Determination of the Exciton Binding Energy in CdSe Quantum Dots, ACS Nano, 2009, 3 (2), pp. 325-330.

10. Jarosz, M. The physics and Chemistry of Transport in CdSe Quantum dot Solids, Ph.D. Thesis, Massachusetts Institute of Technology, USA, 2004. 